\section{EDUCATIONAL POSTERS IMPROVE MANAGEMENT OF STABLE ANGINA IN OUTPATIENTS: A FULL-CYCLE QUALITY IMPROVEMENT PROJECT}

${ }^{1}$ Lawrence O'Leary*, 'Samantha Montandon, ${ }^{2}$ Mohammed Obeidat, ${ }^{3}$ Vasileios Lamprou, ${ }^{1}$ Ursula Doris, ${ }^{1}$ Matthew King, ${ }^{1}$ Som Chuah, ${ }^{1}$ Erwin Rodrigues. ${ }^{1}$ Aintree University Hospital; ${ }^{2}$ Warrington and Halton Hospitals; ${ }^{3}$ Southport and Ormskirk Hospital

\subsection{6/heartjnl-2019-BCS.108}

Introduction Stable angina is an important cause of morbidity affecting a large number of people in the UK. The National Institute for Health and Care Excellence (NICE) provides UK national guidelines on management of stable angina. We explored whether these guidelines were being utilised and the effect on adherence of displaying educational posters that summarise the guidelines.

Methods A retrospective audit conducted at a large UK teaching hospital analysed clinic letters of adult patients with a diagnosis of stable angina attending the cardiology outpatient department over a 1 month period $(n=211)$. Management of patients according to the clinic letters was audited against NICE guidelines.

Educational posters were created that summarised NICE guidelines and encouraged accurate documentation of patients' medications and previous discussions and intolerances to treatments. These posters were displayed in clinic rooms, and their effects on compliance to NICE guidelines were re-audited over a 1 month period $(n=140)$.

Results Comparing the initial audit to the re-audit, the latter demonstrated improvements in the proportion of patients offered a short-acting nitrate $(66 \%$ vs. $81 \%$, $\mathrm{p}=0.0015$ ).

There were also improvements in the proportion of patients offered aspirin ( $83 \%$ vs. 95\%, p=0.0027), excluding those with documented contraindications. There were increases in the proportion of documented interacting medications, previous intolerances and other contraindications to aspirin (9 vs. $27 \%$, $\mathrm{p}=0.000013$ ).

No changes were seen in the proportion of patients offered statins (91 vs. $90 \%, \mathrm{p}=0.80$ ), nor in the proportion of diabetic patients offered an ACE inhibitor (77 vs. 79\%, $\mathrm{p}=0.78)$.

No changes were seen in the proportion of patients offered $\beta$-blockers and/or calcium-channel blockers (91 vs. $90 \%, \mathrm{p}=0.96$ ), but there was a significantly greater proportion of documented previous intolerances or contraindications to these medications in the re-audit (1 vs. $10 \%$, $\mathrm{p}=0.000069$ ).

In the re-audit, a greater proportion of patients on third-line anti-anginal medications (isosorbide mononitrate, ranolazine, ivabradine and/or nicorandil) had revascularisation therapy offered or had a discussion documented that it was deemed inappropriate $(52$ vs. $80 \%, \mathrm{p}=0.00012)$.
Of those whose medication had been changed in the appointment, there were increases in the proportion of patients offered follow up in clinic or by their GP within the recommended 4 weeks $(15$ vs. 29\%, $\mathrm{p}=0.0071)$.

Conclusion This full-cycle quality improvement project shows that displaying educational posters in a cardiology outpatient department is an effective method to improve adherence to NICE guidelines on managing stable angina. Determination of whether these effects reflect true improvements to compliance, or better documentation and communication in clinic letters, is planned.

Conflict of Interest None

\section{GLOBAL AND TEMPORAL TRENDS IN MORTALITY FROM ISCHAEMIC HEART DISEASE: STATISTICS FROM THE WORLD HEALTH ORGANISATION}

Alexandra Nowbar*, Mauro Gitto, James Howard, Darrel Francis, Rasha Al-Lamee. Imperial College London

\subsection{6/heartjnl-2019-BCS.109}

Background Ischaemic heart disease (IHD) has been considered the top cause of mortality globally. However, countries differ in their rates and there have been changes over time.

Methods We analysed mortality data submitted to the World Health Organization (WHO) from 2005 to 2015 by individual countries. We explored patterns in relationships with age, sex and income, and calculated age-standardised mortality rates for each country in addition to crude death rates.

Results Russia, United States and Ukraine had the largest absolute numbers of deaths among the countries that provided data. Mortality from IHD has progressively decreased from 2005 to 2015 (Figure 1). Age-standardised IHD mortality rates per 100,000 people per year were much higher in Ukraine (324) and Kazakhstan (97) than in Brazil (54) and the UK (46), with much less difference in other causes of death (Figure 2). All 4 countries showed a progressive decline in IHD mortality.

Conclusions IHD remains the single largest cause of death in countries of all income groups. Rates are very different between countries and are falling in most countries, indicating great potential for further gains. The political and economic transitions that have taken place in Eastern Europe and Central Asia may have contributed to trends in IHD mortality and risk factor control.

Conflict of Interest nil 